\title{
Indirect NMR spin-spin coupling constants in diatomic alkali halides
}

Michał Jaszuński, Andrej Antušek, Taye B. Demissie, Stanislav Komorovsky, Michal Repisky, and Kenneth Ruud

Citation: The Journal of Chemical Physics 145, 244308 (2016); doi: 10.1063/1.4972892

View online: $\mathrm{http}: / / \mathrm{dx}$. doi.org/10.1063/1.4972892

View Table of Contents: http://aip.scitation.org/toc/jcp/145/24

Published by the American Institute of Physics

\section{Articles you may be interested in}

Hyperfine interactions and internal rotation in methanol

The Journal of Chemical Physics 145, 244301244301 (2016); 10.1063/1.4972004

Nuclei-selected atomic-orbital response-theory formulation for the calculation of NMR shielding tensors using density-fitting

The Journal of Chemical Physics 145, 234108234108 (2016); 10.1063/1.4972212

Constricted variational density functional theory for spatially clearly separated charge-transfer excitations The Journal of Chemical Physics 145, 244108244108 (2016); 10.1063/1.4972231

Theoretical study of actinide monocarbides (ThC, UC, PuC, and AmC)

The Journal of Chemical Physics 145, 244310244310 (2016); 10.1063/1.4972812

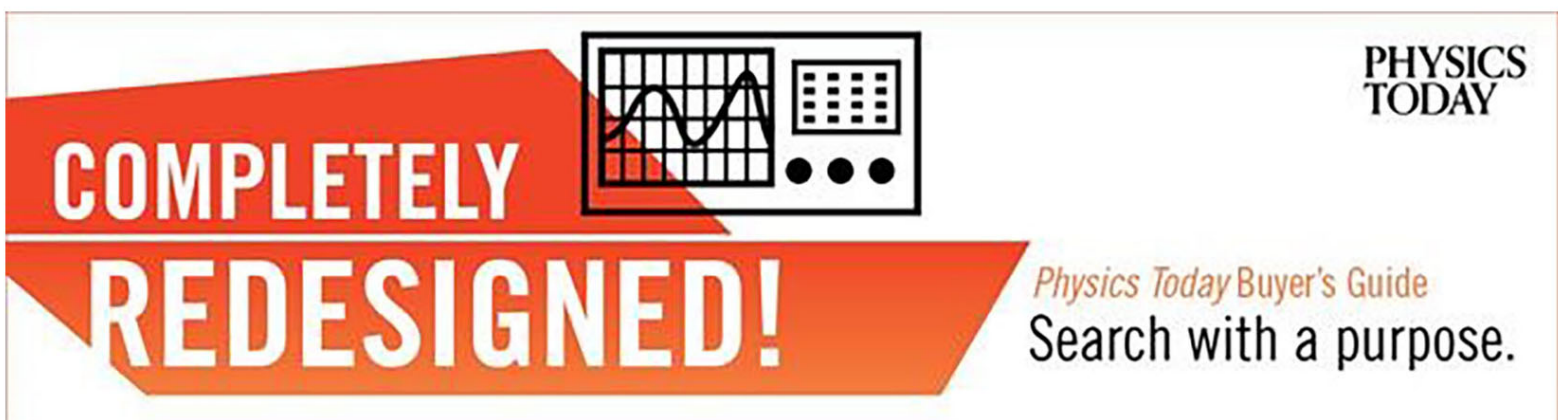




\title{
Indirect NMR spin-spin coupling constants in diatomic alkali halides
}

\author{
Michał Jaszuński, ${ }^{1}$ Andrej Antušek, ${ }^{2}$ Taye B. Demissie, ${ }^{3}$ Stanislav Komorovsky, ${ }^{3}$ \\ Michal Repisky, ${ }^{3}$ and Kenneth Ruud ${ }^{3}$ \\ ${ }^{1}$ Institute of Organic Chemistry, Polish Academy of Sciences, Kasprzaka 44, 01224 Warszawa, Poland \\ ${ }^{2}$ ATRI, Faculty of Materials Science and Technology in Trnava, Slovak University of Technology in Bratislava, \\ Paulinska 16, 91724 Trnava, Slovak Republic \\ ${ }^{3}$ Department of Chemistry, Centre for Theoretical and Computational Chemistry, UiT The Arctic University \\ of Norway, N-9037 Troms $\phi$, Norway
}

(Received 29 September 2016; accepted 10 December 2016; published online 28 December 2016)

\begin{abstract}
We report the Nuclear Magnetic Resonance (NMR) spin-spin coupling constants for diatomic alkali halides $\mathrm{MX}$, where $\mathrm{M}=\mathrm{Li}, \mathrm{Na}, \mathrm{K}, \mathrm{Rb}$, or $\mathrm{Cs}$ and $\mathrm{X}=\mathrm{F}, \mathrm{Cl}, \mathrm{Br}$, or I. The coupling constants are determined by supplementing the non-relativistic coupled-cluster singles-and-doubles (CCSD) values with relativistic corrections evaluated at the four-component density-functional theory (DFT) level. These corrections are calculated as the differences between relativistic and non-relativistic values determined using the PBE0 functional with 50\% exact-exchange admixture. The total coupling constants obtained in this approach are in much better agreement with experiment than the standard relativistic DFT values with 25\% exact-exchange, and are also noticeably better than the relativistic PBE0 results obtained with 50\% exact-exchange. Further improvement is achieved by adding rovibrational corrections, estimated using literature data. Published by AIP Publishing. [http://dx.doi.org/10.1063/1.4972892]
\end{abstract}

\section{INTRODUCTION}

Indirect nuclear spin-spin coupling constant is one of the key Nuclear Magnetic Resonance (NMR) parameters. It provides detailed information about molecular structure-bond distances, angles, and the relative proximity of the interacting nuclei. Isotropic values of the spin-spin coupling constants as well as the individual tensor components can be measured in the condensed phase. More accurate values, not affected by intermolecular interactions, can be determined from gas-phase spectra. Accurate experimental data can be also derived from molecular beam and high-resolution microwave spectra. ${ }^{1}$ Numerous results obtained in this manner are available for diatomic molecules, in particular for alkali halides. ${ }^{2}$ In these gas-phase, low-pressure experiments, the intermolecular effects are negligible, and these results are therefore very important for benchmarking different computational approaches applied to predict and interpret indirect spin-spin coupling constants.

The theoretical calculations of spin-spin coupling constants face two major challenges. The first is that a proper treatment of electron correlation effects is required, and second one has to take into account relativistic effects. The latter problem becomes more pronounced when heavy atoms are present in the molecular systems, as documented by several groups in research articles ${ }^{3-12}$ and reviews. ${ }^{13,14}$ The relativistic effects can be interpreted by applying perturbation theory; ${ }^{15}$ for example, the effects caused by the interplay between the Fermi-contact (FC) interaction of nucleus $\mathrm{K}$ with the spinorbit (SO) interaction generated by the magnetic moment of nucleus $\mathrm{L}$ can be analyzed. ${ }^{16-18}$ On the other hand, to include most of the relevant one- and two-electron relativistic corrections into the calculation of indirect spin-spin coupling constants in a more systematic way, it is convenient to start from two-component ${ }^{19-21}$ or four-component ${ }^{22-25}$ relativistic Hamiltonians. In this way, both scalar and spin-orbit relativistic corrections are included into the wave function variationally and thus relativistic effects to arbitrary order are accounted for. Four-component density-functional theory (DFT) has in recent years reached a level of maturity that makes it an efficient and reliable technique for routine studies of NMR parameters on medium-sized systems. ${ }^{26-28}$ In this work we therefore use the four-component formalism along with a restricted magnetically balanced (RMB) basis for the small component ${ }^{25,29}$ in order to eliminate problems with summations over negativeenergy states and thus the strong basis-set dependence of NMR parameters observed in some early four-component calculations. $^{30}$

The relativistic theory using the RMB formalism for calculating indirect spin-spin coupling constants has been presented and assessed in calculations on $\mathrm{XH}_{4}(\mathrm{X}=\mathrm{C}, \mathrm{Si}, \mathrm{Ge}, \mathrm{Sn})$ by Repisky et al. ${ }^{25}$ Despite these methodological advances, relativistic DFT calculations are not always straightforward. This is, for instance, evident from the difference between spin-spin coupling constants calculated using generalized gradient approximation (GGA) and hybrid functionals. ${ }^{6,10}$ In our recent work on absolute shielding constants, ${ }^{31-35}$ we have shown for various nuclei that both challenges can be overcome and experimental values may be reproduced by combining accurate non-relativistic coupled-cluster results with relativistic corrections obtained from four-component relativistic DFT calculations.

In this work, we present the indirect spin-spin coupling constants of 20 alkali halide diatomic molecules determined using the same approach-by adding relativistic corrections from DFT to the non-relativistic coupled-cluster values. The 
results are compared with available experimental data. We provide new accurate and reliable results for coupling constants that are not known experimentally. We also compare the reduced coupling constants, calculated using different computational approaches, to provide a more detailed comparison of the role of different contributions.

\section{COMPUTATIONAL METHODS}

For the sake of consistency, we present the results for the most abundant isotopic species, that is (the corresponding magnetogyric ratios ${ }^{36}$ are given in parentheses) for ${ }^{7} \mathrm{Li}(10.3977013),{ }^{23} \mathrm{Na}(7.0808493),{ }^{39} \mathrm{~K}$ (1.250 060 8), ${ }^{85} \mathrm{Rb}(2.5927050),{ }^{133} \mathrm{Cs}(3.5332539),{ }^{19} \mathrm{~F}(25.18148)$, ${ }^{35} \mathrm{Cl}(2.624198),{ }^{79} \mathrm{Br}(6.725616)$, and ${ }^{127} \mathrm{I}$ (5.389 573). We recall that for a fixed molecular geometry the coupling constants for other isotopic species can be obtained considering only the changes in the magnetogyric ratios. The molecular geometries are taken from the NIST diatomic spectral database $^{37}$ (mostly based on the compilation by Huber and Herzberg $\left.^{38}\right)$. We have used the following internuclear distances ( $\mathrm{R}_{e}$ in $\AA$ ): LiF: 1.563 864; LiCl: 2.020 673; LiBr: 2.170 427; LiI: 2.391 924; NaF: 1.925 94; NaCl: 2.360 79; NaBr: 2.502 03; NaI: 2.711 45; KF: 2.171 45; KCl: 2.666 65; KBr: 2.820 78; KI: 3.047 84; RbF: 2.270333 ; RbCl: 2.78673 ; RbBr: 2.944 74; RbI: 3.176 879; CsF: 2.345 351; CsCl: 2.906 27; CsBr: 3.072 25; CsI: 3.31519.

At the non-relativistic level we use the coupled-cluster singles-and-doubles (CCSD) model. For the spin-spin coupling constants, the appropriate linear response CCSD formalism has been presented by Auer and Gauss, ${ }^{39}$ and we use the implementation available in the CFOUR program. ${ }^{40}$ There are not too many all-electron basis sets available for all the atoms studied in this work. We shall report the results obtained with the uncontracted ANO-RCC basis sets; ${ }^{41}$ we have also performed test calculations applying the uncontracted AQZP basis ${ }^{42,43}$ and the dyall-cvtz basis set (see below). The results were very similar; therefore at the CCSD level we have not analyzed further the basis set dependence. Test calculations for LiI, $\mathrm{NaF}$, and $\mathrm{RbBr}$ were also performed using the second-order polarization propagator approximation, SOPPA, ${ }^{44}$ (implemented in the Dalton program ${ }^{45}$ ) and the uncontracted AQZP basis set. In all CCSD calculations, point nuclear charge and magnetic moment distributions were used.

The four-component relativistic DFT calculations of the spin-spin coupling constants were done using the DiracCoulomb Hamiltonian with the restricted magnetically balanced condition imposed at the integral level for the coupling of large and small components ${ }^{25}$ and noncollinear GGA theory for the evaluation of DFT kernels. ${ }^{46}$ We have first considered LiI, $\mathrm{NaF}$, and $\mathrm{RbBr}$, studying the performance of the Dirac-Hartree-Fock (DHF) and Dirac-Kohn-Sham (DKS) methods, the latter with both the GGA-type $\mathrm{PBE}^{47}$ and hybridtype $\mathrm{PBE}^{48}$ functionals, by employing Dyall's relativistic all-electron core-valence triple- $\zeta$ basis sets (abbreviated as dyall-cvtz) ${ }^{49-52}$ and quadruple- $\zeta$ basis sets (abbreviated as dyall-cvqz), ${ }^{50-52}$ all in uncontracted form. In all the other fourcomponent DFT calculations we used the dyall-cvtz basis set in uncontracted form. Although they are somewhat smaller
TABLE I. Comparison of isotropic spin-spin coupling constants in LiI, NaF, and $\mathrm{RbBr}$ : benchmarking different computational methods $\left(K\right.$ in $\left.10^{19} \mathrm{~T}^{2} \mathrm{~J}^{-1}\right)$.

\begin{tabular}{|c|c|c|c|}
\hline Methods $^{\mathrm{a}}$ & LiI & $\mathrm{NaF}$ & $\mathrm{RbBr}$ \\
\hline Expt. & $66.39 \pm 0.40^{\mathrm{b}}$ & $58.76 \pm 4.74^{\mathrm{c}}$ & $323.09 \pm 4.34^{\mathrm{c}}$ \\
\hline $\mathrm{NR} / \mathrm{HF}$ & 108.33 & 93.65 & 424.95 \\
\hline NR/PBE & 35.03 & 21.72 & 78.71 \\
\hline NR/PBE0(25\%) & 52.43 & 43.45 & 180.22 \\
\hline NR/PBE0(40\%) & 62.65 & 53.45 & 231.74 \\
\hline NR/PBE0(50\%) & 69.21 & 59.02 & 262.37 \\
\hline NR/PBE0 $(50 \%)^{\mathrm{d}}$ & 70.58 & 60.75 & 265.21 \\
\hline $\mathrm{NR} / \mathrm{SOPPA}^{\mathrm{e}}$ & 73.58 & 52.78 & 291.28 \\
\hline $\mathrm{NR} / \mathrm{CCSD}^{\mathrm{f}}$ & 73.72 & 57.43 & 301.33 \\
\hline DHF & 104.87 & 95.64 & 508.88 \\
\hline DKS/PBE & 23.80 & 23.63 & 96.64 \\
\hline DKS/PBE0(25\%) & 40.82 & 44.85 & 200.62 \\
\hline DKS/PBE0(40\%) & 51.97 & 54.80 & 261.22 \\
\hline DKS/PBE0(50\%) & 59.54 & 60.38 & 298.76 \\
\hline DKS/PBE0 $(50 \%)^{\mathrm{d}}$ & 60.84 & 62.10 & 300.95 \\
\hline SO-ZORA/PBE0 $(25 \%)^{\mathrm{g}}$ & 41.54 & 42.60 & 195.48 \\
\hline SO-ZORA/PBE0 $(40 \%)^{\mathrm{g}}$ & 53.65 & 54.15 & 254.56 \\
\hline SO-ZORA/PBE0 $(50 \%)^{\mathrm{g}}$ & 61.89 & 60.71 & 291.73 \\
\hline
\end{tabular}

${ }^{\mathrm{a}}$ The percentages in parentheses refer to the amount of the exact-exchange admixture (in the standard PBE0 it is $25 \%$ ). Uncontracted dyall-cvtz basis set was used unless otherwise stated.

$\mathrm{b}_{J}=62.44 \pm 0.38 \mathrm{~Hz}$; taken from Ref. 55 .

${ }^{\mathrm{c}} \mathrm{NaF}: J=175.84 \pm 14.18 \mathrm{~Hz}$ and $\mathrm{RbBr}: J=94.56 \pm 1.27 \mathrm{~Hz}$; evaluated for $\mathrm{v}=0, \mathrm{~J}=0$ using the data of Ref. 2.

${ }^{\mathrm{d}}$ Uncontracted dyall-cvqz basis set.

${ }^{\mathrm{e}}$ Uncontracted AQZP basis set.

${ }^{\mathrm{f}}$ Uncontracted ANO-RCC basis set.

$\mathrm{g}_{\mathrm{JCPL}}$ basis set.

than the corresponding ANO-RCC basis sets used in the non-relativistic CCSD approach, Dyall's basis sets have been optimised for four-component calculations. The effect of increasing the size of the basis set from triple- $\zeta$ to quadruple- $\zeta$ on the relativistic corrections is less than $1 \%$ for LiI and $\mathrm{NaF}$ and less than $2 \%$ for $\mathrm{RbBr}$ (see Table I), justifying the use of the dyall-cvtz basis. In order to estimate the relativistic effects, we also performed spin-spin coupling calculations using the same functional and basis sets with an increased speed of light $(100 \times)$ to obtain the non-relativistic values. For the nuclear charge distribution, the finite-size Gaussian-type model was used in the relativistic calculations, whereas the point-type model was used in the non-relativistic calculations. For the magnetic moment, the point-type model was used in all the calculations. All these DFT results were obtained using a development version of the program package ReSpect. ${ }^{53}$ Additional test calculations for $\mathrm{LiI}, \mathrm{NaF}$, and $\mathrm{RbBr}$ were also performed at the SO-ZORA level, using the Amsterdam Density Functional $(\mathrm{ADF})^{54}$ program package employing the $\mathrm{PBE0}^{48}$ functional together with the $\mathrm{JCPL}^{6}$ basis set.

\section{RESULTS AND DISCUSSION}

As a basis for our discussion, we have tested various methods for a small subset of alkali halides: LiI, $\mathrm{NaF}$, and $\mathrm{RbBr}$. A comparison of the results obtained with available experimental spin-spin coupling constants (SSCC) can be made from the data reported in Table I. The non-relativistic (NR) Hartree-Fock (HF) and four-component Dirac-Hartree-Fock 
(DHF) approaches significantly overestimate the experimental data, demonstrating the importance of electron correlation. The correlation effects are described consistently by CCSD, the NR/CCSD values are in good agreement with experiment. The difference is about $5 \mathrm{~Hz}$ for all three molecules, including LiI, suggesting that the relativistic effects are small in this subset of molecules. For these three molecules, we have tested also the performance of the CCSD method with the dyall-cvtz basis set. The results are very close to the tabulated ANO-RCC values, and in particular for the dominant FC terms, the difference is below $1 \%$ for $\mathrm{LiI}$ and $\mathrm{NaF}$, and $<3 \%$ for $\mathrm{RbBr}$, respectively. The non-relativistic SOPPA method gives results that are of slightly poorer agreement with experiment.

The SSCC from non-relativistic DFT with a hybrid functional-usually the method of choice-are shown in Table I and designated as NR/PBE0(25\%) results. They differ significantly from the experimental values, even in the case of the light NaF molecule. The discrepancy is much larger for molecules with heavy elements such as LiI and RbBr; other standard functionals such as BP86 show a similar performance. The four-component relativistic DKS/PBE0 does not significantly improve the agreement; on the contrary, for LiI the result is even worse than that from NR/PBE0. The quality of the PBE0 results can be improved by manipulating the exactexchange admixture in the exchange-correlation functional. In particular, better agreement between the DFT and experimental SSCC of the alkali halides can be obtained with an increased amount of exact exchange in the hybrid functional, as previously observed by Bryce and Autschbach. ${ }^{55}$
As shown in Table I, with the amount of exact-exchange increasing from the standard $25 \%$, the DFT results approach the experimental values. Significantly improved results are indeed obtained with a customized PBE0 (50\%) functional which includes $50 \%$ exact-exchange admixture (instead of the default $25 \%$ in PBE0). However, the improvement is not systematic, DKS/PBE0(50\%) already overshoots the experiment for $\mathrm{NaF}$ whereas the SSCC for LiI is still $10 \%$ lower than experiment. For comparison, we have in Table I also included the results of relativistic SO-ZORA/PBE0 - a two-component relativistic method used in Ref. 55. To summarize the discussion of the results in Table I, it is unlikely that one can obtain reliable SSCC of all the alkali halides by straightforward application of DFT. It seems that alkali halides, although very simple molecules with an ionic bond, are difficult for DFT, which in many other cases yields fairly accurate and reliable SSCC constants (see, e.g., Refs. 56 and 57).

We now turn to a discussion of the CCSD results, as they constitute our best non-relativistic reference (more advanced analytic CC3 methods may become generally available soon; ${ }^{58}$ however, only finite-perturbation calculations at the CC3 level have been described in the literature to date ${ }^{59,60}$ ). The results for all the alkali halides are collected in Table II. The Fermi contact (FC) term is dominant, the paramagnetic spin-orbit (PSO) contribution is significant; there is a very minor contribution from the spindipole (SD) term and practically negligible contribution of the diamagnetic spin-orbit (DSO) term. The total equilibrium non-relativistic $J_{\mathrm{NR}}$ is in very good agreement with experimental SSCC for most of the alkali halides, with the exception

TABLE II. Isotropic SSCC, coupled-cluster $J_{\mathrm{NR}}$, relativistic and vibrational corrections, and the total spin-spin coupling constants (in Hz). ${ }^{\mathrm{a}}$

\begin{tabular}{|c|c|c|c|c|c|c|c|c|c|c|c|}
\hline & \multicolumn{4}{|c|}{$\mathrm{NR} / \mathrm{CCSD}^{\mathrm{b}}$} & \multicolumn{3}{|c|}{$\operatorname{PBE0}(50 \%)$} & \multirow[b]{2}{*}{$J_{\text {eq }}$} & \multirow[b]{2}{*}{$\Delta_{\mathrm{Vib}}$} & \multirow[b]{2}{*}{$J_{\text {total }}$} & \multirow[b]{2}{*}{$J_{\exp }{ }^{\mathrm{c}}$} \\
\hline & $\mathrm{FC}$ & $\mathrm{SD}$ & PSO & $J_{\mathrm{NR}}$ & NR & DKS & $\Delta_{\text {rel }}$ & & & & \\
\hline $\mathrm{LiF}$ & 179.37 & 0.23 & -8.41 & 171.17 & 174.44 & 175.06 & 0.62 & 171.79 & -2.41 & 169.38 & $172.3 \pm 2.6$ \\
\hline $\mathrm{LiCl}$ & 17.62 & -0.05 & -0.57 & 17.00 & 16.61 & 16.73 & 0.12 & 17.12 & 0.33 & 17.45 & $\mathrm{n} / \mathrm{a}$ \\
\hline $\mathrm{LiBr}$ & 68.27 & -0.30 & -2.20 & 65.77 & 58.92 & 57.41 & -1.51 & 64.26 & -0.39 & 63.87 & $60.4 \pm 7.0$ \\
\hline LiI & 71.40 & -0.36 & -1.71 & 69.34 & 65.10 & 56.00 & -9.10 & 60.24 & 2.10 & 62.34 & $62.44 \pm 0.38$ \\
\hline $\mathrm{NaF}$ & 207.74 & 0.61 & -36.47 & 171.87 & 176.62 & 180.69 & 4.07 & 175.94 & 1.06 & 177.00 & $175.84 \pm 14.18^{\mathrm{d}}$ \\
\hline $\mathrm{NaCl}$ & 24.52 & 0.01 & -3.32 & 21.22 & 19.25 & 19.83 & 0.58 & 21.79 & -0.02 & 21.77 & $0 \pm 300,50 \pm 30$ \\
\hline $\mathrm{NaBr}$ & 95.30 & -0.02 & -14.19 & 81.09 & 57.57 & 59.19 & 1.61 & 82.71 & 1.99 & 84.69 & $78 \pm 3$ \\
\hline $\mathrm{NaI}$ & 103.44 & -0.14 & -12.83 & 90.46 & 72.47 & 60.66 & -11.81 & 78.65 & 2.99 & 81.64 & $\mathrm{n} / \mathrm{a}$ \\
\hline $\mathrm{KF}$ & 60.89 & -0.06 & -9.13 & 51.70 & 52.08 & 53.99 & 1.91 & 53.61 & 0.54 & 54.15 & $57.8 \pm 1.3$ \\
\hline $\mathrm{KCl}$ & 6.63 & 0.00 & -1.01 & 5.62 & 5.35 & 5.60 & 0.25 & 5.87 & -0.01 & 5.86 & $9 \pm 6$ \\
\hline $\mathrm{KBr}$ & 27.06 & 0.01 & -4.63 & 22.45 & 18.89 & 19.83 & 0.94 & 23.38 & -0.14 & 23.24 & $21.89 \pm 0.18$ \\
\hline KI & 29.35 & 0.01 & -4.55 & 24.81 & 22.44 & 22.01 & -0.43 & 24.39 & 0.41 & 24.80 & $24.10 \pm 0.29$ \\
\hline $\mathrm{RbF}$ & 238.50 & -0.79 & -31.76 & 205.95 & 207.46 & 235.18 & 27.72 & 233.67 & -0.94 & 232.73 & $236 \pm 0.29$ \\
\hline $\mathrm{RbCl}$ & 25.77 & -0.02 & -3.87 & 21.88 & 21.03 & 23.98 & 2.95 & 24.83 & -0.02 & 24.81 & $25.77 \pm 0.92$ \\
\hline $\mathrm{RbBr}$ & 106.39 & -0.15 & -18.04 & 88.19 & 76.79 & 87.44 & 10.65 & 98.84 & 0.1 & 98.94 & $94.56 \pm 1.27^{\mathrm{d}}$ \\
\hline $\mathrm{RbI}$ & 115.17 & -0.04 & -18.19 & 96.93 & 90.02 & 98.00 & 7.98 & 104.91 & 0.42 & 105.33 & $103.68 \pm 0.37^{d}$ \\
\hline $\mathrm{CsF}$ & 515.29 & -3.02 & -51.68 & 460.59 & 444.15 & 611.97 & 167.82 & 628.41 & -3.14 & 625.27 & $622.94 \pm 0.34$ \\
\hline $\mathrm{CsCl}$ & 53.51 & -0.17 & -7.61 & 45.73 & 43.97 & 59.60 & 15.63 & 61.36 & 0.12 & 61.48 & $60.5 \pm 1.6$ \\
\hline $\mathrm{CsBr}$ & 224.70 & -0.64 & -36.61 & 187.46 & 170.91 & 228.17 & 57.26 & 244.72 & 1.95 & 246.67 & $\mathrm{n} / \mathrm{a}$ \\
\hline CsI & 241.57 & -0.47 & -38.39 & 202.71 & 192.32 & 253.58 & 61.26 & 263.97 & -0.53 & 263.44 & $\mathrm{n} / \mathrm{a}$ \\
\hline
\end{tabular}

${ }^{\mathrm{a}}$ Non-relativistic $J_{\mathrm{NR}}=\mathrm{FC}+\mathrm{SD}+\mathrm{PSO}+\mathrm{DSO}, J_{\mathrm{eq}}=J_{\mathrm{NR}}+\Delta_{\mathrm{rel}}$ are equilibrium SSCC with the relativistic correction $\Delta_{\mathrm{rel}}=\mathrm{DKS} / \mathrm{PBE} 0(50 \%)-\mathrm{NR} / \mathrm{PBE} 0(50 \%) ; J_{\mathrm{total}}=J_{\mathrm{NR}}+\Delta_{\mathrm{rel}}+\Delta_{\mathrm{vib}}$ is the final SSCC, where $\Delta_{\text {vib }}$ are rescaled vibrational corrections taken from Ref. 55 (see the text).

${ }^{\mathrm{b}}$ The DSO contributions are included, they are not listed because all the absolute values are $<0.01 \mathrm{~Hz}$, except for the $\mathrm{LiF}$ value, $-0.015 \mathrm{~Hz}$.

${ }^{\mathrm{c}}$ Reference 55 .

${ }^{\mathrm{d}}$ Evaluated for $\mathrm{v}=0, \mathrm{~J}=0$ using the data of Ref. 2. 
TABLE III. $\chi^{2}$ fit of various theoretical methods to experimental data.

\begin{tabular}{lcrr}
\hline \hline Method & $a$ & \multicolumn{1}{c}{$b$} & \multicolumn{1}{c}{$\chi^{2}$} \\
\hline SO-ZORA/PBE0(25\%) $+\Delta_{\text {vib }}{ }^{\text {a }}$ & 1.01546 & 11.73250 & 5104.16 \\
DKS/PBE0(25\%) & 1.08207 & 13.02494 & 5798.15 \\
DKS/PBE0 $50 \%)$ & 1.01195 & 2.08050 & 408.08 \\
CCSD $+\Delta_{\text {rel }}(25 \%)$ & 1.03371 & -0.86425 & 204.30 \\
CCSD $+\Delta_{\text {rel }}(50 \%)$ & 0.99418 & -0.01308 & 320.74 \\
CCSD $+\Delta_{\text {rel }}(50 \%)+\Delta_{\text {vib }}$ & 0.99982 & -0.50623 & 262.34 \\
\hline \hline
\end{tabular}

${ }^{\text {a }}$ Source data taken from Ref. 55.

of the molecules containing the cesium atom. Next, the relativistic correction $\Delta_{\text {rel }}$ was calculated at the DFT level as the difference between four-component $\mathrm{PBE} 0(50 \%)$ and nonrelativistic PBE0(50\%) values of the SSCC; that is, in both sets of calculations, the exact-exchange admixture in the functional was increased to $50 \%$. The relativistically corrected SSCC equilibrium values, $J_{\text {eq }}$, are in excellent agreement with experimental values. We note that whenever the relativistic corrections are significant, they bring $J_{\text {eq }}$ closer to the experimental value. The tabulated vibrational corrections $\Delta_{\text {vib }}$ were rescaled from the vibrational corrections in Ref. 55. Bryce and Autschbach ${ }^{55}$ have calculated vibrational corrections using hybrid DFT including scalar and spin-orbit relativistic effects and discussed the percentage contribution of these corrections to the equilibrium values. Hence, we used their results with an additional rescaling factor defined by the ratio of our equilibrium value to the corresponding value of Ref. 55. This approach allowed us to circumvent the computationally demanding CCSD calculations of vibrational corrections; most likely the results would not be significantly different. Our final values of $J_{\text {total }}$ are determined by adding the relativistic corrections from $\mathrm{PBE} 0(50 \%)$ to the CCSD equilibrium results, and finally adding the rescaled vibrational corrections.

The accuracy of the different approximations was assessed by fitting a linear function $y=a x+b$ to the pairs of calculated and corresponding experimental spin-spin coupling constants $\left\{J_{\text {calc }}^{i}, J_{\exp }^{i}\right\}_{i}$. All molecules with available experimental data were included in the data set; for $\mathrm{NaCl}$ the more accurate value $50 \pm 30 \mathrm{~Hz}$ was taken. The $a$ and $b$ parameters were found by minimizing the $\chi^{2}(a, b)$ function, ${ }^{61}$

$$
\chi^{2}(a, b)=\sum_{i}\left(\frac{J_{\mathrm{exp}}^{i}-a J_{\text {calc }}^{i}-b}{\sigma_{i}}\right)^{2},
$$

which takes into account the magnitude of experimental error bars $\sigma_{i}$ in the fit (in the ideal case of perfect agreement between experiment and theoretical values $a=1, b=0$, and $\left.\chi^{2} \Rightarrow 0\right)$.

The $\chi^{2}$ fits of the SSCC data for various theoretical models are collected in Table III. The comparison shows the poor performance of DFT hybrid functionals with standard $25 \%$ admixture of exact-exchange, $\chi^{2}$ is very high for SO-ZORA/PBE0 and DKS/PBE0. Increasing the exactexchange admixture to $50 \%$ leads to statistically significant improvement of the agreement between experimental and theoretical data. In most cases the prediction becomes very satisfactory; however, it is not satisfactory for $\mathrm{NaBr}$ and $\mathrm{NaI}$ (for $\mathrm{NaBr}$, the DKS/PBE0(50\%) value differs by $26 \%$ from the experimental one), for no obvious reason. This behaviour of DKS/PBE0(50\%) deteriorates the general reliability of this approximation.

Theoretical models based on the CCSD method with various relativistic corrections provide very good agreement with experimental data, as shown by $\chi^{2}$ values in the range $\approx 200-300$. Although CCSD with relativistic corrections based on standard PBE0 $(25 \%)$ provides the lowest $\chi^{2}$ value, it has the worst $a$ parameter among the CCSD-based models. It appears that the best model is provided by a method which places the theoretical predictions as close as possible to the $a=1(y=x)$ line in the $J_{\text {calc }}$ vs. $J_{\text {exp }}$ plot (see Fig. 1). CCSD combined with relativistic corrections calculated from the PBE0(50\%) functional and including vibrational corrections fulfills this requirement almost perfectly with $a=0.99982$ and a very small shift $b \approx-0.5 \mathrm{~Hz}$. We note that a $\chi^{2}$ difference of about 50 corresponds to a difference of $2.1 \mathrm{~Hz}$ in a single experimental SSCC value with a declared error bar of $0.3 \mathrm{~Hz}$; $\chi^{2}$ variations in the range 200-300 are not really meaningful. For a visual comparison, the fits of our best model CCSD $+\Delta_{\text {rel }}(50 \%)+\Delta_{\text {vib }}$ and the SO-ZORA/PBE0 $(25 \%)+\Delta_{\text {vib }}$ as a model of lower reliability are depicted in Fig. 1. We observe that for all molecules the points are systematically shifted to the $y=x$ line when we consider the CCSD $+\Delta_{\text {rel }}(50 \%)+\Delta_{\text {vib }}$ model instead of the SO-ZORA/PBE0 $(25 \%)+\Delta_{\text {vib }}$ model. We observed a similar effect with DKS/PBE0(25\%).

The magnitudes of the SSCC do not illustrate the general trends of indirect spin-spin coupling interactions mediated by electrons because they depend on the magnetic dipole moments of the interacting nuclei. The role of the electronic part of this phenomenon can be analyzed by considering

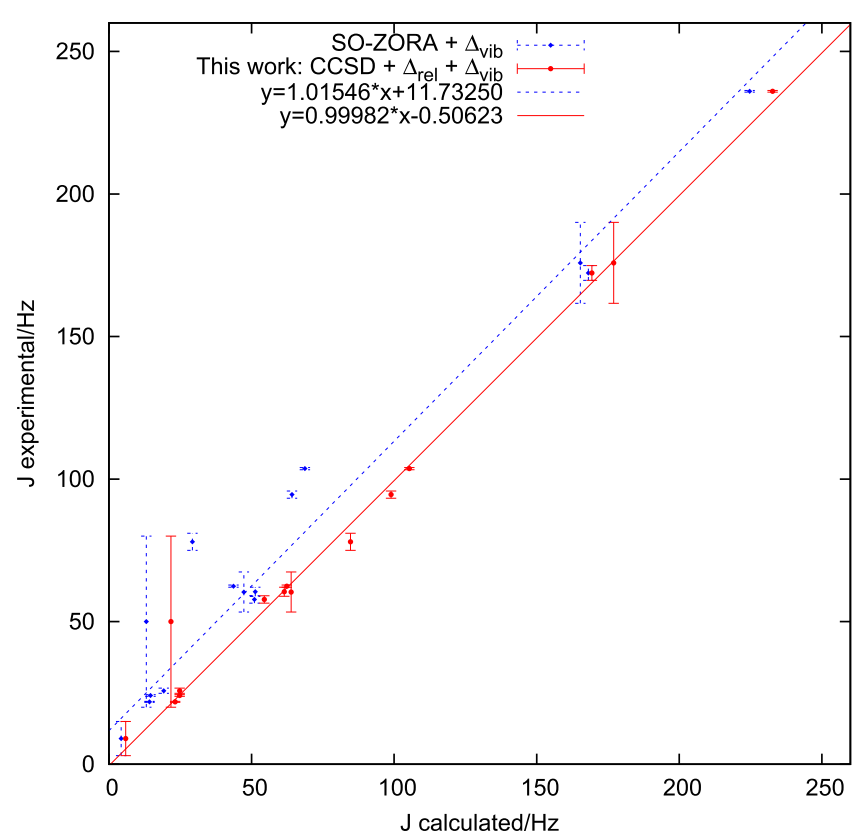

FIG. 1. Comparison of the calculated and experimental $J$ values (SO-ZORA results taken from Ref. 55). The data sets are fitted by a linear function minimizing $\chi^{2}$ (see the text for details). CsF data are included in the fit, but they are not depicted due to scale. 
TABLE IV. Comparison of non-relativistic, relativistic, and total reduced spin-spin coupling constants $K$ (in $10^{19} \mathrm{~T}^{2} \mathrm{~J}^{-1}$ ). The percentages denote HF contributions to the PBE0 functional.

\begin{tabular}{|c|c|c|c|c|c|c|c|c|c|c|c|}
\hline & \multicolumn{2}{|c|}{ SO-ZORA/PBE0 ${ }^{\mathrm{a}}$} & \multicolumn{2}{|c|}{ NR/PBE0 } & \multicolumn{2}{|c|}{ DKS/PBE0 } & \multirow{2}{*}{$\begin{array}{c}\Delta_{\mathrm{SC}}{ }^{\mathrm{b}} \\
50 \%\end{array}$} & \multirow{2}{*}{$\begin{array}{l}\Delta_{\mathrm{SO}^{\mathrm{c}}} \\
50 \%\end{array}$} & \multirow{2}{*}{$\begin{array}{l}\Delta_{\text {rel }} \\
50 \%\end{array}$} & \multirow[b]{2}{*}{ CCSD } & \multirow[b]{2}{*}{$K_{\text {total }}{ }^{\mathrm{d}}$} \\
\hline & $25 \%$ & $50 \%$ & $25 \%$ & $50 \%$ & $25 \%$ & $50 \%$ & & & & & \\
\hline $\mathrm{LiF}$ & 38.8 & 42.5 & 35.69 & 39.70 & 35.79 & 39.84 & 0.11 & 0.03 & 0.14 & 38.95 & 39.09 \\
\hline $\mathrm{LiCl}$ & 31.5 & 36.2 & 31.53 & 36.28 & 31.69 & 36.53 & 0.02 & 0.23 & 0.26 & 37.13 & 37.39 \\
\hline $\mathrm{LiBr}$ & 40.6 & 51.8 & 39.09 & 50.20 & 37.16 & 48.91 & -3.70 & 2.42 & -1.28 & 56.04 & 54.75 \\
\hline LiI & 44.9 & 64.0 & 52.43 & 69.21 & 40.82 & 59.54 & -18.28 & 8.61 & -9.67 & 73.72 & 64.06 \\
\hline $\mathrm{NaF}$ & 54.9 & 69.3 & 43.45 & 59.02 & 44.85 & 60.38 & 0.59 & 0.77 & 1.36 & 57.43 & 58.79 \\
\hline $\mathrm{NaCl}$ & 42.0 & 62.4 & 42.45 & 61.73 & 44.45 & 63.58 & -0.52 & 2.37 & 1.84 & 68.03 & 69.87 \\
\hline $\mathrm{NaBr}$ & 36.0 & 81.6 & 23.77 & 72.02 & 29.24 & 74.06 & -20.28 & 22.31 & 2.03 & 101.45 & 103.48 \\
\hline $\mathrm{NaI}$ & 31.7 & 102.0 & 37.75 & 113.14 & 29.21 & 94.70 & -91.16 & 72.71 & -18.45 & 141.23 & 122.85 \\
\hline $\mathrm{KF}$ & 95.4 & 107.9 & 85.32 & 98.57 & 86.27 & 102.18 & 3.53 & 0.08 & 3.61 & 96.61 & 100.24 \\
\hline $\mathrm{KCl}$ & 78.8 & 99.1 & 78.52 & 97.22 & 82.15 & 101.67 & 2.89 & 1.56 & 4.45 & 102.14 & 106.59 \\
\hline $\mathrm{KBr}$ & 101.4 & 149.6 & 85.37 & 133.88 & 91.42 & 140.53 & -10.84 & 17.49 & 6.65 & 159.07 & 165.72 \\
\hline KI & 127.3 & 208.0 & 122.75 & 198.40 & 119.08 & 194.60 & -64.08 & 60.28 & -3.80 & 219.43 & 215.65 \\
\hline $\mathrm{RbF}$ & 205.8 & 228.6 & 168.76 & 189.32 & 188.47 & 214.62 & 28.04 & -2.74 & 25.30 & 187.94 & 213.23 \\
\hline $\mathrm{RbCl}$ & 168.6 & 208.9 & 152.53 & 184.15 & 171.07 & 209.97 & 25.42 & 0.39 & 25.82 & 191.63 & 217.44 \\
\hline $\mathrm{RbBr}$ & 218.9 & 314.5 & 180.22 & 262.37 & 200.62 & 298.76 & 7.83 & 28.57 & 36.40 & 301.34 & 337.72 \\
\hline $\mathrm{RbI}$ & 292.0 & 448.2 & 255.39 & 383.82 & 266.52 & 417.85 & -75.66 & 109.69 & 34.03 & 413.31 & 447.36 \\
\hline $\mathrm{CsF}$ & 405.0 & 430.4 & 282.19 & 297.42 & 378.57 & 409.80 & 128.00 & -15.62 & 112.38 & 308.43 & 420.76 \\
\hline $\mathrm{CsCl}$ & 328.8 & 384.9 & 248.03 & 282.54 & 325.86 & 382.96 & 111.02 & -10.60 & 100.42 & 293.85 & 394.22 \\
\hline $\mathrm{CsBr}$ & 471.6 & 610.7 & 341.63 & 428.52 & 426.02 & 572.09 & 135.39 & 8.17 & 143.56 & 470.00 & 613.51 \\
\hline CsI & 630.9 & 861.5 & 467.69 & 601.71 & 557.92 & 793.39 & 104.97 & 86.71 & 191.68 & 634.23 & 825.87 \\
\hline
\end{tabular}

${ }^{\mathrm{a}}$ Reference 55 .

${ }^{\mathrm{b}} \Delta_{\mathrm{SC}}-$ scalar relativistic correction.

${ }^{\mathrm{c}} \Delta_{\mathrm{SO}}-$ spin-orbit relativistic correction.

${ }^{\mathrm{d}} K_{\text {total }}=K_{\mathrm{CCSD}}+\Delta_{\text {rel }}(50 \%)$.

instead the reduced spin-spin coupling constants, $K$. Nonrelativistic CCSD and relativistically corrected CCSD + DKS/ PBE0(50\%) $K$ values are collected in Table IV (showing also the partition of the relativistic correction into scalar and spinorbit contributions) and depicted, separately for the fluorides, chlorides, bromides, and iodides series, in Fig. 2, revealing smooth trends. The general features are as follows: first, $K$ increases with the atomic number of the alkali atom in the molecule, and second, significant relativistic effects are introduced by the heavy alkali metal atoms $\mathrm{Rb}$ and $\mathrm{Cs}$. For the
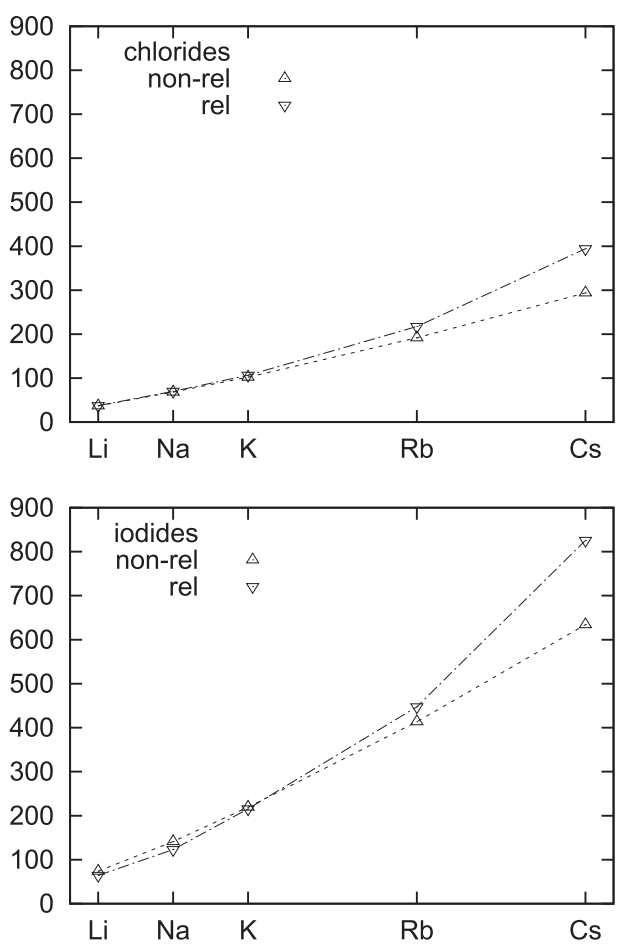

FIG. 2. Relativistic and non-relativistic $K$ (in $10^{19} \mathrm{~T}^{2} \mathrm{~J}^{-1}$ ). 
other cases, the relativistic effects are small; surprisingly, the presence of an iodine atom does not introduce strong overall relativistic effects, which is a result of substantial cancellation of scalar and spin-orbit relativistic corrections (with the exception of CsI, see Table IV). This highlights the importance of taking both scalar and spin-orbit effects into account in relativistic calculations of spin-spin coupling constants.

\section{CONCLUSIONS}

We have demonstrated that the spin-spin coupling constants of alkali halides calculated using CCSD with relativistic corrections estimated at the DFT level utilizing a PBE0 functional with 50\% exact-exchange admixture and with vibrational corrections are in excellent agreement with available gas-phase experimental data. Consequently, we expect that our predictions for the SSCC for the $\mathrm{LiCl}, \mathrm{NaI}, \mathrm{CsBr}$, and CsI molecules, for which experimental data are missing, and for $\mathrm{NaCl}$ (with large experimental error bar) are reliable. The agreement of our results with experiment confirms the assumed additivity of non-relativistic CCSD values and relativistic corrections determined within the applied DKS approach. This is advantageous since no practical implementation of a wavefunction based method for the calculation of NMR parameters with simultaneous high-level treatment of electron correlation and relativistic effects is presently available. The computational recipe presented in this work thus represents a useful strategy for the accurate theoretical prediction of NMR parameters. In the case of the SSCC of the alkali halides studied here, the results are much more reliable than those obtained using standard DFT methods. However, the performance of such computational recipes may depend on the specific properties of the molecules studied, and the universality of the approach presented here should be subject to further investigation.

\section{ACKNOWLEDGMENTS}

We acknowledge financial support from Slovak Grant Nos. APVV-15-0105 and VEGA 1/0279/16, Polish National Science Centre on the basis of the Decision No. DEC2014/15/B/ST4/05039 and the Research Council of Norway through a Centre of Excellence Grant (Grant No. 179568/V30) and two project grants (Grant Nos. 214095 and 177558). We have used the computational resources of The Norwegian Supercomputing program NOTUR (Grant No. NN4654K), HPC Cluster of Slovak University of Technology and Computing Center of the Slovak Academy of Sciences (Project Nos. ITMS 26230120002 and 26210120002). Funding by the ERDF-Research and Development Operational Programme under the project "University Scientific Park Campus MTF STU-CAMBO" ITMS: No. 26220220179 is also acknowledged.

${ }^{1}$ J. Vaara, J. Jokisaari, R. E. Wasylishen, and D. L. Bryce, Prog. Nucl. Magn. Reson. Spectrosc. 41, 233 (2002).

${ }^{2}$ A. Faucher and R. E. Wasylishen, in Gas Phase $N M R$, edited by K. Jackowski and M. Jaszuński (The Royal Society of Chemistry, 2016), Chap. 2, pp. 52-94.

${ }^{3}$ P. Pyykkö and L. Wiesenfeld, Mol. Phys. 43, 557 (1981).

${ }^{4}$ J. Jokisaari and J. Autschbach, Phys. Chem. Chem. Phys. 5, 4551 (2003).

${ }^{5}$ A. Bagno and M. Bonchio, Magn. Reson. Chem. 42, S79 (2004).
${ }^{6}$ S. Moncho and J. Autschbach, J. Chem. Theory Comput. 6, 223 (2010).

${ }^{7}$ I. L. Rusakova, Yu. Yu. Rusakov, and L. B. Krivdin, Magn. Reson. Chem. 52, 500 (2014).

${ }^{8}$ A. Wodyński and M. Pecul, J. Chem. Phys. 140, 024319 (2014).

${ }^{9}$ M. Kauch and M. Pecul, J. Phys. Chem. A 118, 4471 (2014).

${ }^{10}$ T. B. Demissie, N. Kostenko, S. Komorovsky, M. Repisky, J. Isaksson, A. Bayer, and K. Ruud, J. Phys. Org. Chem. 28, 723 (2015).

${ }^{11}$ I. L. Rusakova, Yu. Yu. Rusakov, and L. B. Krivdin, Magn. Reson. Chem. 54, 39 (2016).

${ }^{12}$ C. A. Giménez, A. F. Maldonado, and G. A. Aucar, Theor. Chem. Acc. 135, 201 (2016).

${ }^{13} \mathrm{~J}$. Autschbach and T. Ziegler, in Calculation of NMR and EPR Parameters. Theory and Applications, edited by M. Kaupp, M. Bühl, and V. G. Malkin (Wiley-VCH, Weinheim, 2004), Chap. 15, pp. 249-264.

${ }^{14}$ I. L. Rusakova, Yu. Yu. Rusakov, and L. B. Krivdin, Russ. Chem. Rev. 85, 356 (2016).

${ }^{15} \mathrm{~J}$. Vaara, P. Manninen, and P. Lantto, in Calculation of NMR and EPR Parameters. Theory and Applications, edited by M. Kaupp, M. Bühl, and V. G. Malkin (Wiley-VCH, Weinheim, 2004), Chap. 13, pp. 209-226.

${ }^{16} \mathrm{~S}$. Kirpekar, H. J. Aa. Jensen, and J. Oddershede, Theor. Chim. Acta 95, 35 (1997).

${ }^{17}$ S. Kirpekar and S. P. A. Sauer, Theor. Chem. Acc. 103, 146 (1999).

${ }^{18}$ J. Vaara, K. Ruud, and O. Vahtras, J. Comput. Chem. 20, 1314 (1999).

${ }^{19}$ J. Autschbach and T. Ziegler, J. Chem. Phys. 113, 936 (2000).

${ }^{20}$ J. Autschbach and T. Ziegler, J. Chem. Phys. 113, 9410 (2000).

${ }^{21}$ J. I. Melo, M. C. Ruiz de Azúa, J. E. Peralta, and G. E. Scuseria, J. Chem. Phys. 123, 204112 (2005).

${ }^{22}$ L. Visscher, T. Enevoldsen, T. Saue, H. J. Aa. Jensen, and J. Oddershede, J. Comput. Chem. 20, 1262 (1999).

${ }^{23}$ G. A. Aucar and J. Oddershede, Int. J. Quantum Chem. 47, 425 (1993).

${ }^{24}$ G. A. Aucar, T. Saue, L. Visscher, and H. J. Aa. Jensen, J. Chem. Phys. 110, 6208 (1999)

${ }^{25}$ M. Repisky, S. Komorovsky, O. L. Malkina, and V. G. Malkin, Chem. Phys. 356, 236 (2009).

${ }^{26}$ P. Hrobarik, V. Hrobarikova, F. Meier, M. Repisky, S. Komorovsky, and M. Kaupp, J. Phys. Chem. A 115, 5654 (2011).

${ }^{27}$ G. Casella, A. Bagno, S. Komorovsky, M. Repisky, and G. Saielli, Chem. Eur. J. 21, 18834 (2015).

${ }^{28}$ J. Vicha, R. Marek, and M. Straka, Inorg. Chem. 55, 1770 (2016).

${ }^{29}$ S. Komorovsky, M. Repisky, O. L. Malkina, V. G. Malkin, I. Malkin Ondik, and M. Kaupp, J. Chem. Phys. 128, 104101 (2008).

${ }^{30}$ J. Vaara and P. Pyykkö, J. Chem. Phys. 118, 2973 (2003).

${ }^{31}$ M. Jaszuński, M. Repisky, T. B. Demissie, S. Komorovsky, E. Malkin, K. Ruud, P. Garbacz, K. Jackowski, and W. Makulski, J. Chem. Phys. 139, 234302 (2013).

${ }^{32}$ K. Ruud, T. B. Demissie, and M. Jaszuński, J. Chem. Phys. 140, 194308 (2014).

${ }^{33}$ M. Jaszuński, T. B. Demissie, and K. Ruud, J. Phys. Chem. A 118, 9588 (2014).

${ }^{34}$ T. B. Demissie, M. Jaszuński, E. Malkin, S. Komorovský, and K. Ruud, Mol. Phys. 113, 1576 (2015).

${ }^{35}$ T. B. Demissie, M. Jaszuński, S. Komorovsky, M. Repisky, and K. Ruud, J. Chem. Phys. 143, 164311 (2015).

${ }^{36}$ R. K. Harris, E. D. Becker, S. M. Cabral de Menezes, R. Goodfellow, and P. Granger, Pure Appl. Chem. 73, 1795 (2001), reprinted in Magn. Reson. Chem. 40, 489-505 (2002).

${ }^{37}$ See http://physics.nist.gov/cgi-bin/MolSpec/diperiodic.pl for NIST diatomic spectral database.

${ }^{38}$ K. P. Huber and G. Herzberg, Molecular Spectra and Molecular Structure. IV. Constants of Diatomic Molecules (Van Nostrand Reinhold, New York, 1979).

${ }^{39}$ A. A. Auer and J. Gauss, J. Chem. Phys. 115, 1619 (2001).

${ }^{40}$ CFOUR, a quantum chemical program package written by J. F. Stanton, J. Gauss, M. E. Harding, and P. G. Szalay with contributions from A. A. Auer, R. J. Bartlett, U. Benedikt, C. Berger, D. E. Bernholdt, J. Bomble, L. Cheng, O. Christiansen, M. Heckert, O. Heun, C. Huber, T.-C. Jagau, D. Jonsson, J. Jusélius, K. Klein, W. J. Lauderdale, D. A. Matthews, T. Metzroth, L. A. Mück, D. P. O'Neill, D. R. Price, E. Prochnow, C. Puzzarini, K. Ruud, F. Schiffmann, W. Schwalbach, C. Simmons, S. Stopkowicz, A. Tajti, J. Vázquez, F. Wang, and J. D. Watts and the integral packages MOLECULE (J. Almlöf and P. R. Taylor), PROPS (P. R. Taylor), ABACUS (T. Helgaker, H. J. Aa. Jensen, P. Jørgensen, and J. Olsen), and ECP routines by A. V. Mitin and C. van Wüllen. For the current version, see http://www.cfour.de. 
${ }^{41}$ B. O. Roos, R. Lindh, P.-Å. Malmqvist, V. Veryazov, and P.-O. Widmark, J. Phys. Chem. A 108, 2851 (2004).

${ }^{42}$ P. A. Fantin, P. L. Barbieri, A. C. Neto, and F. E. Jorge, J. Mol. Struct.: THEOCHEM 810, 103 (2007).

${ }^{43}$ L. S. C. Martins, F. A. L. de Souza, G. A. Ceolin, F. E. Jorge, R. C. de Berredo, and C. T. Campos, Comput. Theor. Chem. 1013, 62 (2013).

${ }^{44}$ T. Enevoldsen, J. Oddershede, and S. P. A. Sauer, Theor. Chem. Acc. 100, 275 (1998).

${ }^{45}$ K. Aidas, C. Angeli, K. L. Bak, V. Bakken, R. Bast, L. Boman, O. Christiansen, R. Cimiraglia, S. Coriani, P. Dahle, E. K. Dalskov, U. Ekström, T. Enevoldsen, J. J. Eriksen, P. Ettenhuber, B. Fernandez, L. Ferrighi, H. Fliegl, L. Frediani, K. Hald, A. Halkier, C. Hättig, H. Heiberg, T. Helgaker, A. C. Hennum, H. Hettema, E. Hjertenæs, S. Høst, I.-M. Høyvik, M. F. Iozzi, B. Jansik, H. J. Aa. Jensen, D. Jonsson, P. Jørgensen, J. Kauczor, S. Kirpekar, T. Kjærgaard, W. Klopper, S. Knecht, R. Kobayashi, H. Koch, J. Kongsted, A. Krapp, K. Kristensen, A. Ligabue, O. B. Lutnæs, J. I. Melo, K. V. Mikkelsen, R. H. Myhre, C. Neiss, C. B. Nielsen, P. Norman, J. Olsen, J. M. H. Olsen, A. Osted, M. J. Packer, F. Pawlowski, T. B. Pedersen, P. F. Provasi, S. Reine, Z. Rinkevicius, T. A. Ruden, K. Ruud, V. Rybkin, P. Salek, C. C. M. Samson, A. S. de Meras, T. Saue, S. P. A. Sauer, B. Schimmelpfennig, K. Sneskov, A. H. Steindal, K. O. Sylvester-Hvid, P. R. Taylor, A. M. Teale, E. I. Tellgren, D. P. Tew, A. J. Thorvaldsen, L. Thøgersen, O. Vahtras, M. A. Watson, D. J. D. Wilson, M. Ziolkowski, and H. Ågren, Wiley Interdiscip. Rev.: Comput. Mol. Sci. 4, 269 (2014); see also, Dalton, a Molecular Electronic Structure Program, Release DALTON2013.0, 2013, see http://daltonprogram.org.

${ }^{46}$ S. Komorovsky, M. Repisky, E. Malkin, T. B. Demissie, and K. Ruud, J. Chem. Theory Comput. 11, 3729 (2015).

${ }^{47}$ J. P. Perdew, K. Burke, and M. Ernzerhof, Phys. Rev. Lett. 77, 3865 (1996).

${ }^{48}$ C. Adamo and V. Barone, J. Chem. Phys. 110, 6158 (1999).

${ }^{49}$ K. G. Dyall, Theor. Chem. Acc. 108, 335 (2002); Erratum 109, 284 (2003).

${ }^{50}$ K. G. Dyall, Theor. Chem. Acc. 115, 441 (2006), basis sets available from the Dirac web site, http://dirac.chem.sdu.dk.
${ }^{51}$ K. G. Dyall, J. Phys. Chem. A 113, 12638 (2009).

${ }^{52} \mathrm{~K}$. G. Dyall, private communication (2012).

${ }^{53}$ ReSpect, version 4.0.0—Relativistic Spectroscopy DFT program of authors M. Repisky, S. Komorovsky, V. G. Malkin, O. L. Malkina, M. Kaupp, and K. Ruud, with contributions from R. Bast, U. Ekström, M. Kadek, S. Knecht, L. Konecny, I. Malkin-Ondik, and E. Malkin, 2016, see http://www.respectprogram.org.

${ }^{54}$ E. J. Baerends, J. Autschbach, A. Berces, F. M. Bickelhaupt, C. Bo, P. M. Boerrigter, L. Cavallo, D. P. Chong, L. Deng, R. M. Dickson, D. E. Ellis, M. van Faassen, L. Fischer, T. H. Fan, C. F. Guerra, S. J. A. van Gisbergen, J. A. Groeneveld, O. V. Gritsenko, M. Gruning, F. E. Harris, P. van den Hoek, C. R. Jacob, H. Jacobsen, L. Jensen, G. van Kessel, F. Kootstra, E. van Lenthe, D. A. McCormack, A. Michalak, J. Neugebauer, V. P. Osinga, S. Patchkovskii, P. H. T. Philipsen, D. Post, C. C. Pye, W. Ravenek, P. Ros, P. R. T. Schipper, G. Schreckenbach, J. G. Snijders, M. Sola, M. Swart, D. Swerhone, G. teVelde, P. Vernooijs, L. Versluis, L. Visscher, O. Visser, F. Wang, T. A. Wesolowski, E. van Wezenbeek, G. Wiesenekker, S. Wolff, T. Woo, A. Yakovlev, and T. Ziegler, ADF2014.01, SCM, Theoretical Chemistry, Vrije Universiteit, Amsterdam, The Netherlands, 2014, http://www.scm.com.

${ }^{55}$ D. L. Bryce and J. Autschbach, Can. J. Chem. 87, 927 (2009).

${ }^{56}$ T. Helgaker, M. Jaszuński, and M. Pecul, Progr. Nucl. Magn. Reson. Spectrosc. 53, 249 (2008).

${ }^{57}$ High Resolution NMR Spectroscopy: Understanding Molecules and Their Electronic Structures, edited by R. H. Contreras (Elsevier, Amsterdam, 2013).

${ }^{58}$ R. Faber, Ph.D. thesis, University of Copenhagen, 2016.

${ }^{59}$ A. A. Auer and J. Gauss, Chem. Phys. 356, 7 (2001).

${ }^{60}$ R. Faber and S. P. A. Sauer, Phys. Chem. Chem. Phys. 14, 16440 (2012).

${ }^{61}$ J. R. Taylor, An Introduction to Error Analysis. The Study of Uncertainties in Physical Measurements, 2nd ed. (University Science Books, Sausalito, California, 1997). 